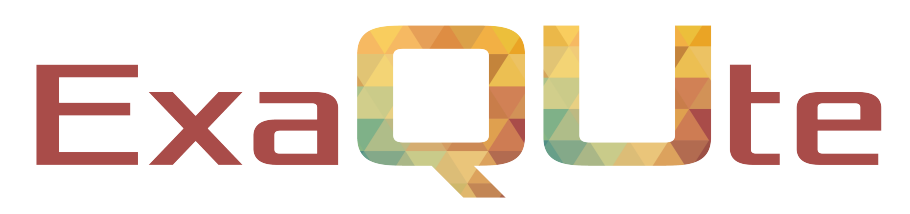

\title{
D5.2 Release of ExaQUte MLMC Python engine
}

\section{Document information table}

\begin{tabular}{|l|l|}
\hline Contract number: & 800898 \\
\hline Project acronym: & ExaQUte \\
\hline Project Coordinator: & CIMNE \\
\hline Document Responsible Partner: & EPFL \\
\hline Deliverable Type: & Other (software) \\
\hline Dissemination Level: & PUblic \\
\hline Related WP \& Task: & WP5 task 5.2 \\
\hline Status: & Approved \\
\hline
\end{tabular}

\begin{tabular}{|c|c|}
\hline 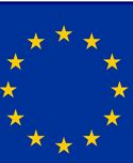 & $\begin{array}{l}\text { This project has received funding from } \\
\text { the European Union's Horizon } 2020 \\
\text { research and innovation programme } \\
\text { under grant agreement No } \mathbf{8 0 0 8 9 8}\end{array}$ \\
\hline
\end{tabular}




\section{Authoring}

\begin{tabular}{|c|c|c|c|c|}
\hline \multicolumn{5}{|c|}{ Prepared by EPFL, CIMNE and BSC } \\
\hline Authors & Partner & Modified & Version & Comments \\
\hline Ramon Amela & $\mathrm{BSC}$ & \multirow{7}{*}{ All } & \multirow{7}{*}{1.0 .0} & $\begin{array}{l}\text { PyCOMPSs } \\
\text { expertise }\end{array}$ \\
\hline Quentin Ayoul-Guilmard & \multirow{2}{*}{ EPFL } & & & \multirow{3}{*}{ Development } \\
\hline Sundar Ganesh & & & & \\
\hline Riccardo Tosi & CIMNE & & & \\
\hline Rosa M. Badia & $\mathrm{BSC}$ & & & \multirow{3}{*}{ Supervision } \\
\hline Fabio Nobile & EPFL & & & \\
\hline Riccardo Rossi & CIMNE & & & \\
\hline
\end{tabular}

\section{Change Log}

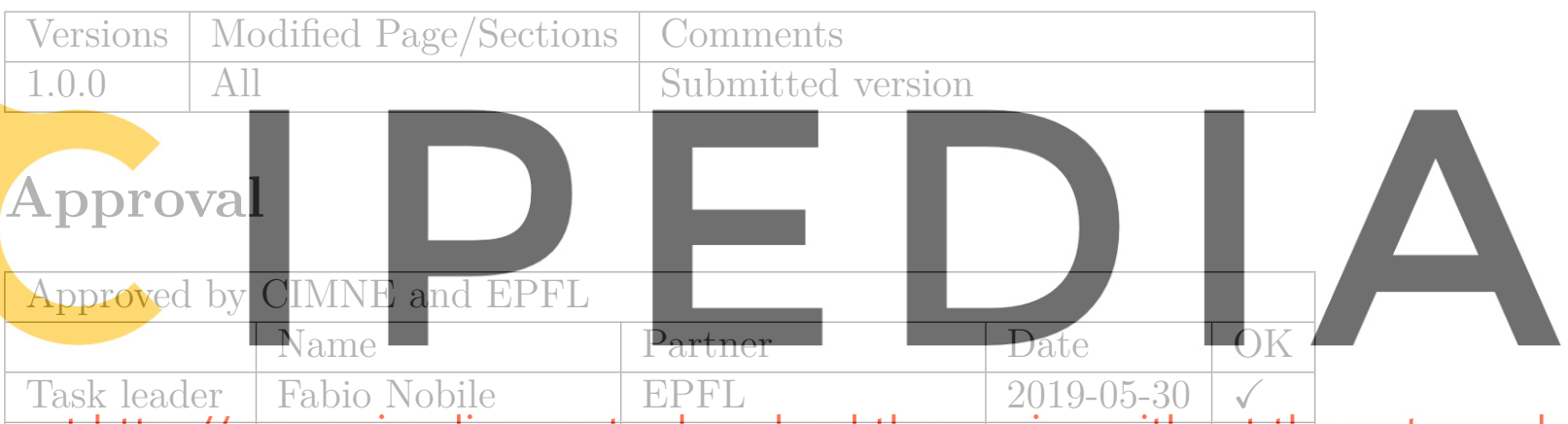

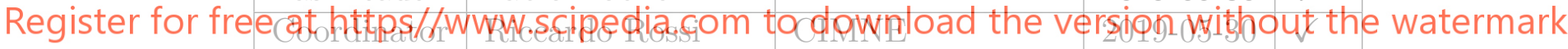

Page 2 of 9 


\section{Executive summary}

In this deliverable, the ExaQUte XMC library is introduced. This report is meant to serve as a supplement to the publicly release of the library. In the following sections, the ExaQUte XMC library is described along with its current and future capabilities. The structure of the library, along with its dynamic import mechanism, are described using samples of code. The algorithms behind the example files supplied with the public release are explained in detail as well.
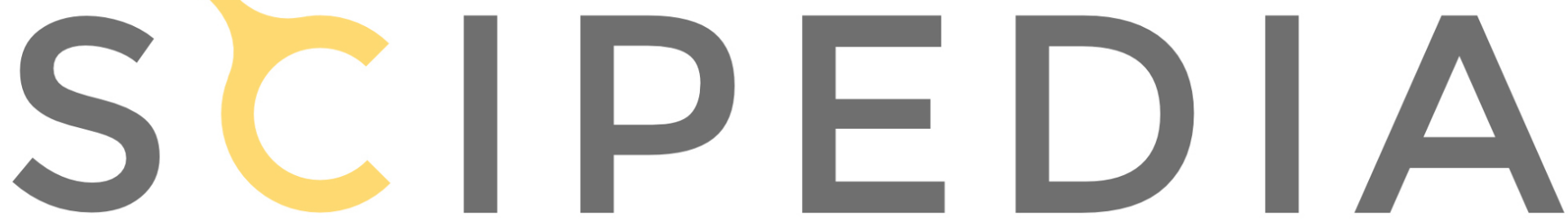

Register for free at https//www.scipedia.com to download the version without the watermark

Page 3 of 9 


\section{Contents}

1 Introduction $\quad 5$

2 ExaQUte XMC library structure 5

$\begin{array}{lll}3 & \text { Instructions to run tutorial files } & 7\end{array}$

4 Tutorial file and algorithm description $\quad 7$

4.1 Simple Monte Carlo . . . . . . . . . . . . . . . . . . . . 7

4.2 Adaptive Monte Carlo ................... . . 7

4.3 Simple Multi-Level Monte Carlo . . . . . . . . . . . . . 8

4.4 Adaptive Multi-Level Monte Carlo . . . . . . . . . . . . . . . 8
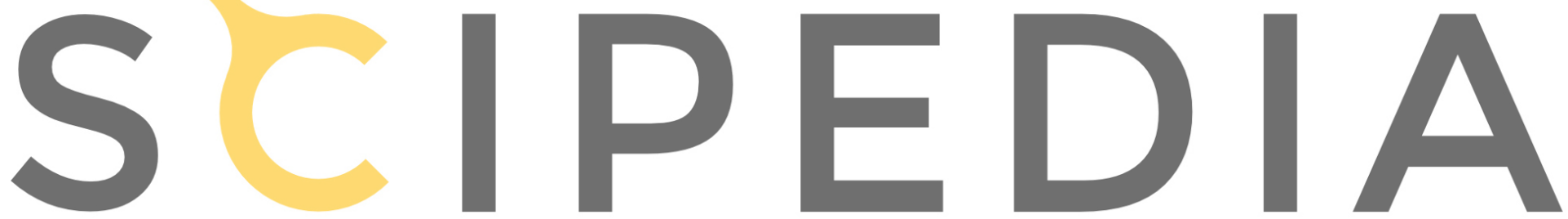

Register for free at https//www.scipedia.com to download the version without the watermark 


\section{Introduction}

The ExaQUte Xmc library [released as 1] offers the user the ability to carry out uncertainty quantification simulations using a multitude of Monte Carlo algorithms. Users of the library will eventually be able to select one of many pre-programmed algorithms such as

1. simple Monte Carlo;

2. adaptive Monte Carlo;

3. simple multi-level Monte Carlo;

4. adaptive multi-level Monte Carlo;

5. continuation multi-level Monte Carlo;

6. multi-index Monte Carlo;
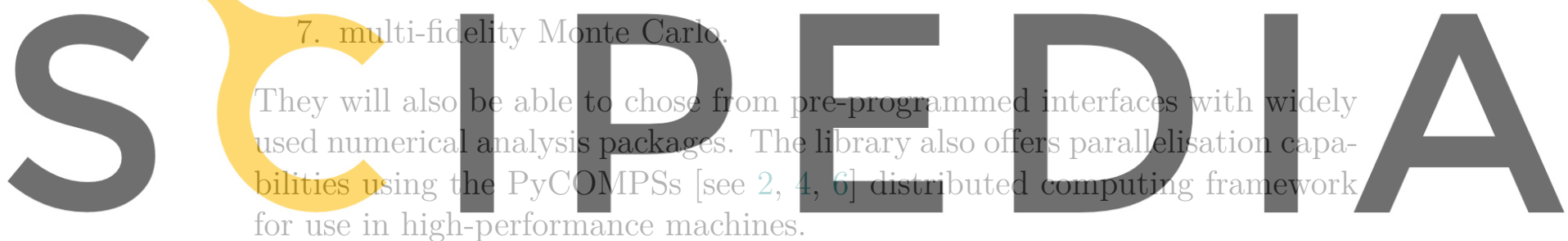

Register for free at https liby is also prgsrammed in a modular way that allows users to construct thelr own $X$ Monte Carlo (XMC) algorithm from several building

block functions. It also allows them to write custom-interfaces easily for their own numerical analysis packages such as custom-finite element analysis or computational fluid dynamics libraries.

\section{ExaQUte XMC library structure}

The ExaQUte XMc library is organised as follows. The global directory xmc is a python package that contains all the packages, subpackages and modules of the library. Within this folder, each file contains the definition of one class. Every file is treated as a module that can be imported. If the class is called ClassName, the file is named className.py

Each class contains multiple types of members. They are as follows

1. Instances of classes

2. Instances of function definition objects 
3. Variables and other data structures

4. Method definitions

The instantiation of function objects is used to enable a function to have multiple definitions. For example, we require that the optimalIndexSet method of the HierarchyOptimiser class to be called as optimalIndexSet(), but to evaluate different expressions for the optimal number of levels based on the type of XMC algorithm used.

In this way the user can simply specify the type of algorithm they would like to run and the definition of optimalIndexSet automatically changes based on this, without any further intervention. This is achieved by instantiating optimalIndexSet dynamically to a specific function definition through the constructor of the HierarchyOptimiser class as follows.

\# hierarchyOptimiser.py

class Hierarchy0ptimiser():

def __init__(self, **keywordArgs):
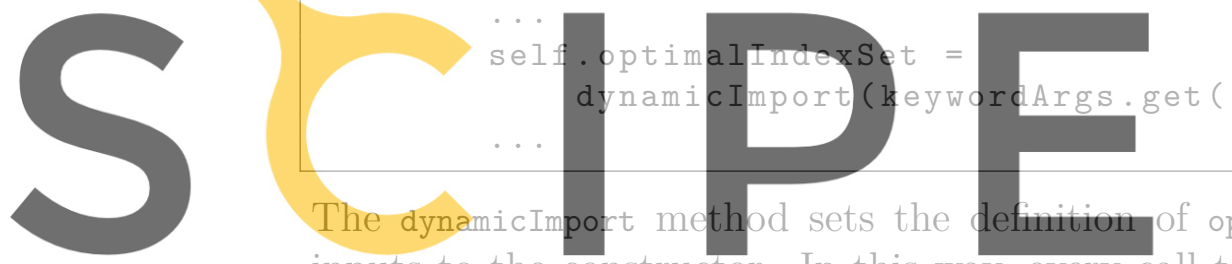

inputs to the constructor. In this way, every call
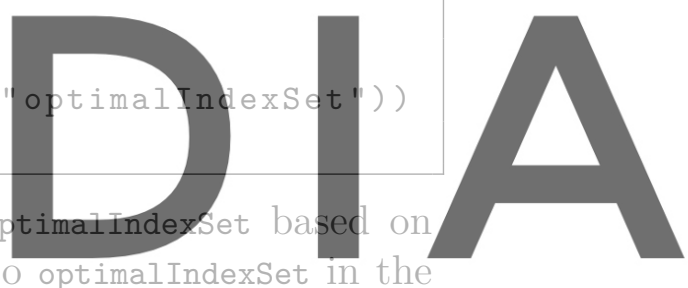

Register for free at https//w.ww. scipedia.com to dow.nload the version without the watermark it is hence implied that every "general method such as optimalindexset

will have a corresponding list of specific definitions, one of which is selected at runtime during the construction of the class containing the method.

To organise this, every className.py file has a corresponding folder by the name methodDefs_className. Inside this folder, there are multiple files named generalMethod.py, one for each member of ClassName that is a function object instance of the class. Within each generalmethod.py, there are a list of definitions as follows -

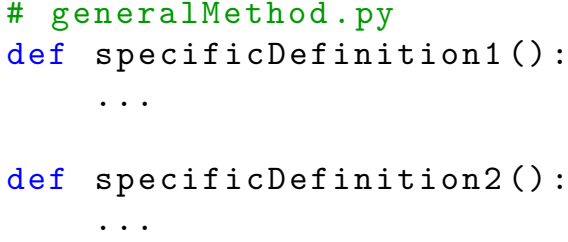

For the example in this section, the instantiation then occurs as follows.

\# elsewhere.py

if (xmcAlgorithmType == "xmcAlgorithm1"): 


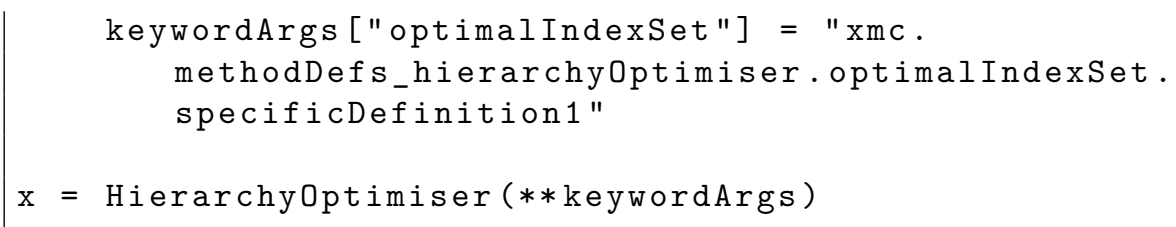

\section{Instructions to run tutorial files}

All tutorial files are to be run as follows

\$ python3 example_*.py
No additional arguments are required. If the user has the PyCOMPSs
scheduling tool installed and the imports in the required files appropriately
set, then the example files can be run with the following command.
\$ runcompss - options=value /global/path/to/example_*.py

However, the user must note that the appropriate imports have to be changed
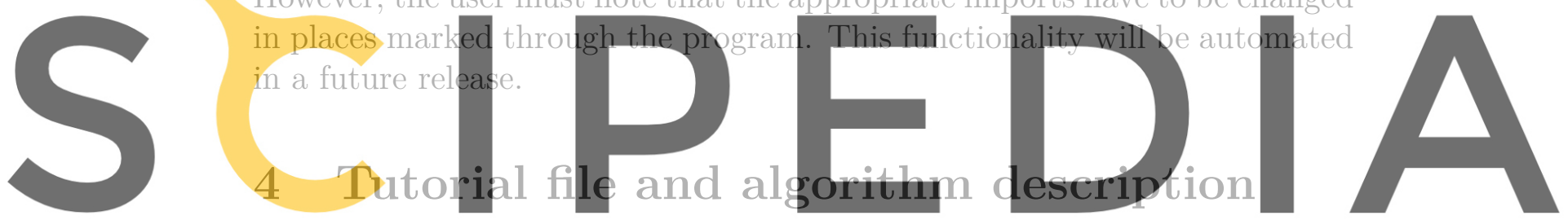
Register for free at https//www. scipedia.com to download the version without the watermark same algorithm as the corresponding example_*.py, but a partial differential equation is solved for the quantity of interest in the Kratos solver [released as 5] instead of a simple random number generator.

\subsection{Simple Monte Carlo}

example_mc.py contains a simple Monte Carlo algorithm that estimates the mean of a standard normal variable. The algorithm begins with a prescribed number of samples. It estimates the mean and the error in the mean estimate. If the error does not satisfy a prescribed tolerance, it doubles the number of samples and repeats the process until convergence.

\subsection{Adaptive Monte Carlo}

example_amc.py contains an adaptive Monte Carlo algorithm that estimates the mean of a standard normal variable. The algorithm begins with a prescribed number of samples. It estimates the mean and the error in the mean 
estimate. If the error does not satisfy a final tolerance, it then computes a new number of samples that will be required to satisfy the tolerance requirement based on the assumption of asymptotic normality, and repeats this procedure until convergence.

\subsection{Simple Multi-Level Monte Carlo}

example_mlmc.py contains a simple Multi-Level Monte Carlo algorithm that estimates the mean of a standard normal variable. A hierarchy of normal random generators whose, the mean and variance of the differences of whose samples decay geometrically with increasing level towards 0 , are used to generate samples. The algorithm begins with a prescribed number of samples and levels. It estimates the mean and the error in the mean estimate. If the error does not satisfy a prescribed tolerance, it increases the number of levels by one, doubles the number of samples in all existing levels, and adds a default number of samples to the new level. This process is repeated until convergence.
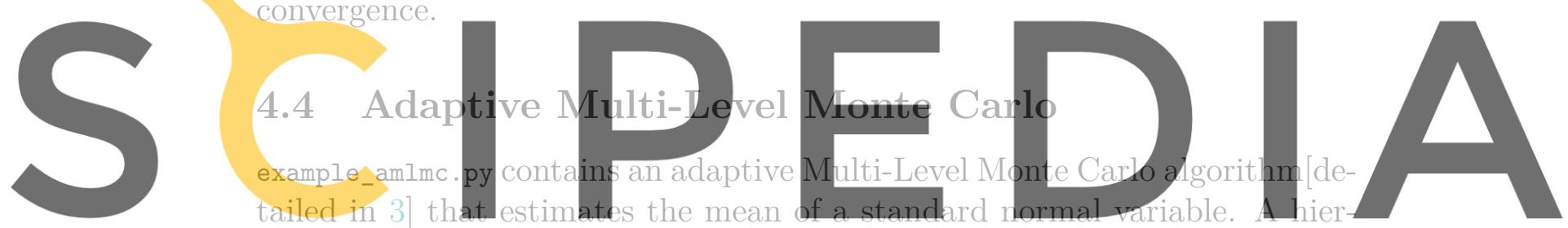

archy of normal random generators whose, the mean and variance of the dif-

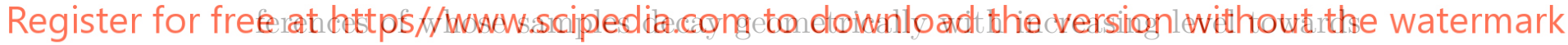

0 , are used to generate samples. The algorithm begins with a prescribed number of samples and levels. It estimates the mean and the error in the mean estimate. It then fits geometric models for the bias, variance and cost across levels on the computed data. If the error does not satisfy a prescribed tolerance, it increases the number of levels by one and computes the costoptimal number of samples for each level using level-wise bias, variance and cost estimates as well as the models for these three quantities. to the new level. This process is repeated until convergence. 


\section{References}

[1] Ramon Amela, Quentin Ayoul-Guilmard, Rosa M Badia, Sundar Ganesh, Fabio Nobile, Riccardo Rossi and Riccardo Tosi. ExaQUte XMC. Comp. software. Version 1.0.0. ExaQUte consortium, May 2019. DOI: 10.5281/ zenodo. 3235833.

[2] Rosa M. Badia, J. Conejero, C. Diaz, J. Ejarque, D. Lezzi, F. Lordan, C. Ramon-Cortes and R. Sirvent. 'COMP Superscalar, an interoperable programming framework'. In: SoftwareX 3-4 (Dec. 2015). DOI: 10 . $1016 / j$. softx. 2015.10.004.

[3] Michael B. Giles. 'Multilevel Monte Carlo methods'. In: Acta Numerica 24 (2015), pp. 259-328. DOI: 10.1017/S096249291500001X.

4] F. Lordan et al. 'ServiceSs: an interoperable programming framework for the Cloud'. In: Journal of Grid Computing 12 (1 Mar. 2014). DoI: $10.1007 /$ s $10723-013-9272-5$.
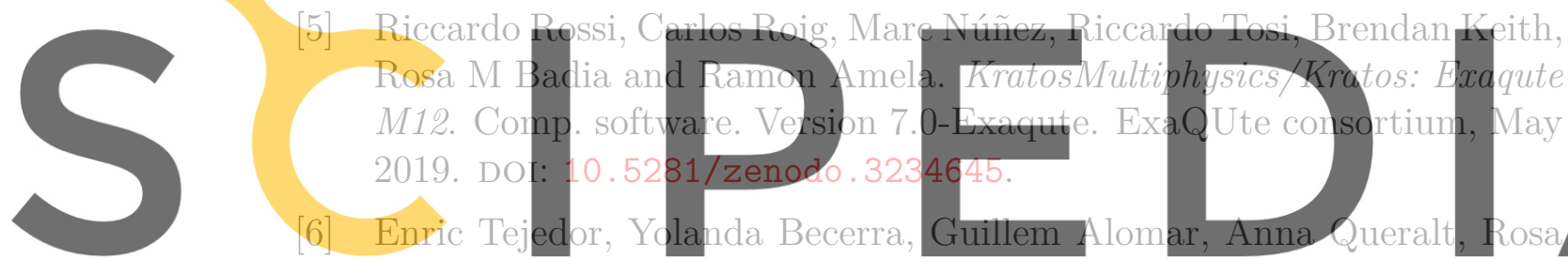

M. Badia, Jordi Torres, Toni Cortes and Jesús Labarta. 'PyCOMPSs:

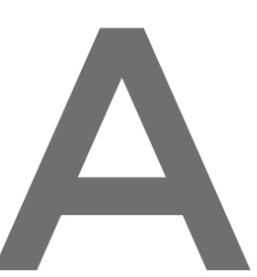

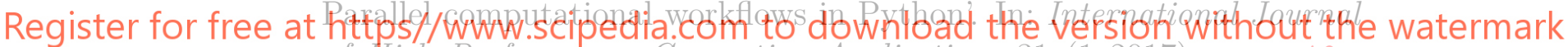
of High Performance Computing Applications 31 (1 2017). DOI: 10 . 